\title{
Percepções sobre qualidade de serviços que atendem à saúde da mulher
}

\author{
Perceptions on the quality of services relating to women's healthcare
}

Christiane Santiago Maia ${ }^{1}$

Daniel Roberto Coradi de Freitas ${ }^{2}$

Dirce Guilhem ${ }^{3}$

Anamaria Ferreira Azevedo ${ }^{4}$
${ }^{1}$ Faculdade de Ciências da Saúde, Universidade de Brasília. AOS 04, bloco B, apto. 423, Área Octogonal. 70660-042 Brasília DF. csmaia2@gmail.com ${ }^{2}$ Gerência Geral de Sangue, Outros Tecidos, Células e Órgãos, Agência Nacional de Vigilância Sanitária. ${ }^{3}$ Departamento de Enfermagem, Faculdade de Ciências da Saúde, Universidade de Brasília.

${ }^{4}$ Secretaria de Estado de Saúde do Distrito Federal.
Abstract The scope of this work is to identify the characteristics relating to the quality of women's healthcare services from the perspective of the sanitary vigilance professionals (Visa) and women's healthcare coordinators (CSM) in the municipalities. It is a study with a qualitative approach that was carried out using semi-structured interviews. Three Brazilian state capitals were selected and three individuals in each capital were interviewed, namely a Visa healthcare service chief, as well as a technician, and a CSM coordinator. The content analysis technique was adopted to organize the data and the answers were grouped into the healthcare service evaluation topics based on Donabedian's model, i.e. structure, processes, and outcomes. Visa workers reported aspects related to the structure of health services, such as equipment and qualified human resources more frequently. The Visa chief mentioned aspects related to structure and processes, such as prioritization in attendance. The CWH coordinator examined all three categories, especially processes. Analysis of interviews points to a potential distortion in evaluation by results. The study highlights the need for joint action between the surveillance and assistance sectors to improve the quality of services. Key words Quality of healthcare, Sanitary vigilance, Women's health
Resumo O objetivo deste trabalho é identificar as características relacionadas à qualidade em serviços que atendem à saúde da mulher na perspectiva de profissionais de vigilância sanitária (Visa) e de coordenadores de saúde da mulher (CSM) de municípios. Trata-se de um estudo de abordagem qualitativa, efetuado por meio de entrevistas semiestruturadas. Foram selecionadas três capitais brasileiras e entrevistados três sujeitos de cada capital - um responsável e um técnico da área de Visa em serviços de saúde e um CSM. A análise das entrevistas foi realizada pela técnica de análise de conteúdo, organizando as respostas em categorias temáticas baseadas no modelo de avaliação dos serviços de saúde de Donabedian: estrutura, processos e resultados. Os técnicos de Visa relataram com maior frequência aspectos relacionados à estrutura dos serviços de saúde, como equipamentos e recursos humanos qualificados. Os responsáveis pela Visa citaram aspectos relacionados à estrutura e aos processos como priorização no atendimento. Os CSM abordaram as três categorias, com destaque para os processos. As entrevistas apontam para um possível prejuízo na avaliação por resultados. O estudo reforça a necessidade de atuação conjunta entre os setores de vigilância e assistência, a fim de promover a qualidade nos serviços.

Palavras-chave Qualidade da assistência à saúde, Vigilância sanitária, Saúde da mulher 


\section{Introdução}

A vigilância sanitária (Visa) pode ser considerada a forma mais complexa de existência da saúde pública. Suas ações, eminentemente preventivas, percorrem todas as práticas médico-sanitárias: promoção, proteção, recuperação e reabilitação da saúde ${ }^{1}$. Pode ser definida como espaço de intervenção do Estado, com a propriedade - por suas funções e instrumentos - de trabalhar no sentido de adequar o sistema produtivo de bens e serviços de interesse sanitário, bem como os ambientes, às demandas sociais de saúde - para os indivíduos e para a coletividade - e às necessidades do sistema de saúde $e^{2}$.

Observa-se que a atual sociedade de consumo impõe o aumento e variação no campo da produção, além da criação incessante da necessidade de consumo para satisfazer às exigências do modo de produção capitalista. Dessa forma, gera constantes riscos à saúde individual e coletiva, fundamentando-se, assim, a necessidade de regulação das relações de produção-consumo ${ }^{3}$. A Visa está inserida nessa lógica de mercado, com o objetivo de eliminar, diminuir ou prevenir riscos à saúde e de intervir nos problemas sanitários decorrentes do meio ambiente, da produção e circulação de bens e da prestação de serviços de interesse da saúde $e^{4}$. Sua atuação reconhece a vulnerabilidade do consumidor e constitui-se em importante ferramenta para o controle social ${ }^{1}$.

Os serviços de saúde podem representar fator de risco aos seus usuários e aos profissionais envolvidos, em decorrência da utilização de procedimentos cada vez mais invasivos e de drogas potentes e mais tóxicas, bem como da incorporação de formas complexas de estruturação e de gestão das organizações 5 .

Diante desse quadro, a área de Visa de serviços de saúde busca a proteção da saúde da população ${ }^{6}$, prevenindo e controlando os riscos e eventos adversos relacionados à prestação de serviços de saúde. As inovações tecnológicas, embora signifiquem avanços, podem também gerar danos à saúde, quando não monitoradas adequadamente. Por isso, a qualidade do atendimento à população está intrinsecamente relacionada à monitoração dos riscos ${ }^{7}$. Nesse sentido, a Visa de serviços de saúde estabelece práticas direcionadas à melhoria da qualidade da atenção à saúde, seja normalizando os procedimentos, seja adotando medidas (inspeções, monitoramento e avaliações, entre outras) e fazendo os prestadores cumprirem condições técnicas minimamente necessárias ao funcionamento dos serviços ${ }^{6}$.
A qualidade dos serviços de saúde configurase hoje em um imperativo técnico e social ${ }^{8}$. Os patamares de exigências, por parte da população, de serviços seguros, eficazes e de qualidade elevaram-se, o que demanda do Estado cada vez mais ações nesse sentido?.

A definição de qualidade apresenta-se de forma multidimensional ${ }^{10}$. No entanto, essas diferentes facetas podem ser sintetizadas em alguns pontos comuns: satisfação das necessidades e expectativas dos clientes; conformidade com as especificações; adequações de uso; zero defeito; fazer mais, melhor e mais rápido; e melhor relação custo versus benefício ${ }^{11}$.

No campo da saúde, qualidade é o produto social expresso por expectativas sobre as relações entre clientes e prestadores de serviços e as formas como são legitimados os papéis no sistema de saúde ${ }^{11}$. Assim, o controle da qualidade reflete a forma como são compartilhadas as responsabilidades públicas e individuais e o grau de preocupação com o direito de acesso ao serviço pela população, bem como a melhoria da saúde pessoal e coletiva ${ }^{12}$.

O quadro conceitual mais utilizado para a avaliação da qualidade dos serviços de saúde é, sem dúvida, o estabelecido por Donabedian ${ }^{13}$. Para o autor, a qualidade não se constitui atributo abstrato e deve ser construída por meio dos sete pilares: eficácia, efetividade, eficiência, otimização, aceitabilidade, legitimidade e equidade ${ }^{13}$. O modelo de análise proposto por Donabedian, que está baseado na avaliação de estrutura, processo e resultado, é amplamente aceito e divulgado em virtude de sua compatibilidade e utilidade para os programas de saúde por contemplar sua lógica de funcionamento: recursos, organização, atividades, serviços e efeitos ${ }^{14}$.

A estrutura trata de características mais estáveis, incluindo informações sobre recursos materiais (como instalações e equipamentos), humanos (número e qualificação) e estrutura organizacional (critérios de operação, sistemas de avaliação etc ${ }^{15}$.). Quando se trata de processo, objetiva-se analisar o "fazer" dos profissionais de saúde, na condução dos cuidados e na interação com os pacientes ${ }^{15}$. Enfim, o resultado voltase ao estudo dos efeitos e consequências das intervenções, utilizando taxas, indicadores, parâmetros de saúde e de satisfação da clientela ${ }^{15}$.

Os dados que compõem os instrumentos utilizados para a apreensão da qualidade dizem respeito às condições estruturais (recursos financeiros, humanos, físicos, organizacionais, entre outros), informações relativas aos processos que 
definem essas práticas (como consultas, internações, cirurgias e exames) e medidas que permitam a mensuração de resultados dessas práticas (por exemplo: mortalidade, morbidade, medidas de qualidade de vida, notificações, tempo de internação $)^{16}$.

O déficit na qualidade dos serviços de saúde de países em desenvolvimento é entendido como desafio-chave ao alcance dos Objetivos de Desenvolvimento do Milênio, definidos pela Organização das Nações Unidas $(\mathrm{ONU})^{17}$, entre os quais está a melhoria da saúde materna. A literatura brasileira também evidencia a importância de serviços de saúde de qualidade para a mulher ${ }^{18}$. A qualificação da atenção consta nos objetivos do Plano Nacional de Políticas para as $\mathrm{Mu}$ lheres, junto com a ampliação do acesso e humanização da atenção ${ }^{19}$.

A história demonstra que a busca das mulheres pelos serviços de saúde está marcada, com frequência, por discriminação e frustrações que repercutem em tensão e mal-estar psicofísico ${ }^{20}$. Os problemas abordados em estudos incluem imperícia e negligência médica, deficiências nas condições estruturais referentes à organização dos serviços de saúde que atendem à mulher ${ }^{21}$, pouca atenção recebida no pré-natal, descaso com os familiares das pacientes, falta de humanização no atendimento e de apoio às mulheres após o parto $^{22}$ e ausência de integração na rede de assistência à mulher no ciclo gravídico-puerperal ${ }^{23}$. Além disso, a incerteza das mulheres quanto ao acesso à instituição hospitalar, bem como o desconhecimento da dinâmica institucional e dos profissionais envolvidos no seu atendimento, acarretam às usuárias um alto grau de estresse ${ }^{23}$.

Muitos dos problemas atuais de saúde da mulher estão relacionados à qualidade da atenção, evidenciando a urgência em se utilizar e integrar todos os campos do saber da saúde que podem colaborar no enfrentamento desse desafio, entre os quais está a Visa ${ }^{24}$.

Para o entendimento do que seja considerado um serviço com qualidade, deve-se partir da premissa de que os critérios de qualidade não são definitivos ou absolutos, uma vez que mudanças na evolução do conhecimento e da dinâmica social exigem novos olhares avaliativos ${ }^{25}$. No atual contexto político, torna-se imprescindível que a prestação de serviços de saúde considere pressupostos constantes na Política Nacional de Atenção Integral à Saúde da Mulher, entre os quais destacamos: disponibilidade de recursos tecnológicos e uso apropriado; estabelecimento de mecanismos de avaliação continuada dos serviços e do desempenho dos profissionais; e análise de indicadores que permitam aos gestores monitorar o impacto das ações sobre os problemas tratados e a redefinição de estratégias ${ }^{19}$.

Tais elementos reforçam a necessidade de que a compreensão da qualidade nos serviços seja baseada em critérios constantes em dimensões tanto de estrutura como de processo e resultado. A complementaridade desses três elementos fortalece o processo avaliativo, com maior possibilidade de serem estabelecidos nexos causais ${ }^{26}$.

Espera-se, ainda, que o serviço atenda às necessidades de saúde definidas tecnicamente, contemplando promoção, prevenção, tratamento e reabilitação ${ }^{26}$. A atenção à saúde deve otimizar os efeitos desejáveis e minimizar os indesejáveis, correspondendo às expectativas e necessidades dos usuários ${ }^{25}$.

O movimento mundial da qualidade e segurança no campo da saúde vem lançando mão de um vasto número de métodos. Busca-se, ultimamente, a integração entre esses instrumentos para projetos específicos ${ }^{17}$. Algumas das razões para a diversidade de abordagens na avaliação da qualidade na saúde incluem a procedência dos avaliadores e a função da avaliação ${ }^{14}$. Quando a avaliação é realizada de forma ética, útil e tecnicamente adequada, reduz incertezas, melhora a efetividade das ações, propicia a tomada de decisões relevantes e subsidia a correção de rumos e a reorientação de estratégias de ação de determinada proposta ${ }^{27}$.

Esse aspecto subjetivo da avaliação da qualidade tem sido pouco explorado e guarda, certamente, em seus meandros, muitos aspectos a serem desvelados, já que pertence ao plano das nuanças, do profundo, do particular ${ }^{10}$. Nessa linha, compreende-se que as percepções dos atores envolvidos com a qualidade dos serviços relacionam-se com suas experiências ${ }^{10}$, o que repercute nas intervenções para a promoção da qualidade.

Considerando que no campo teórico da Visa e da saúde da mulher o tema da qualidade dos serviços mostra-se relevante e direciona as práticas nas secretarias de saúde municipais (lócus de materialização das políticas de saúde), o objetivo deste trabalho é identificar as características relacionadas à qualidade em serviços que atendem à saúde da mulher na perspectiva de profissionais de Visa de serviços de saúde e coordenadores de saúde da mulher (CSM).

Pretende-se, pois, analisar como essa questão é vislumbrada por profissionais de áreas com esse compromisso comum, mas pertencentes a campos distintos da saúde. A apropriação desses en- 
tendimentos sobre a qualidade não objetiva a determinação de qual abordagem seja mais ou menos apropriada, mas sim a expressão dos valores que permeiam os projetos de saúde municipais.

\section{Métodos}

Trata-se de um estudo exploratório, de abordagem qualitativa, efetuado por meio de entrevistas semiestruturadas. No campo da saúde, o debate do "qualitativo" mostra-se apropriado a estudos sobre organização, administração e avaliação dos serviços e sistemas de saúde ${ }^{28}$.

Os critérios utilizados para a seleção dos municípios foram serem capitais de estado, em diferentes regiões. As três cidades selecionadas estão localizadas nas regiões Norte, Nordeste e Sul e constituem uma amostra de conveniência. Buscou-se, desta maneira, apreender dados de municípios com diferenças regionais, mas que tivessem características comuns às capitais. Não são identificadas as cidades nas quais ocorreu a coleta de dados como forma de promover a confidencialidade dos entrevistados.

Previamente ao trabalho de campo, foi efetuado contato telefônico para verificação do interesse em participar do estudo e agendamento das entrevistas com os sujeitos. Após a realização da coleta de dados nas três capitais, propositalmente pertencentes a distintas regiões, observou-se o início da repetição dos discursos. Esse fator foi decisivo para o encerramento da fase de campo e início da análise dos dados, pois demonstrou a consistência e a relevância do que seria aprofundado na discussão dos achados ${ }^{29}$.

Cada município possuía um responsável pela área de Visa de serviços de saúde e um CSM. Entrevistou-se, também, um técnico de Visa sugerido pelo responsável da área de Visa em serviços de saúde. O grupo estudado é formado, portanto, por nove indivíduos: três responsáveis e três técnicos da área de Visa em serviços de saúde e três CSM. Outro pré-requisito para participação na pesquisa foi a assinatura do Termo de Consentimento Livre e Esclarecido (TCLE). O TCLE foi elaborado com linguagem acessível, contendo as informações relacionadas ao processo da pesquisa. Foi garantida a confidencialidade das informações coletadas.

O roteiro de entrevista foi empregado como forma de facilitar a explicitação das percepções dos informantes, tendo-se o cuidado de não configurá-lo em elemento engessador do diálogo. Constituiu-se de questões abertas, que tratavam de aspectos relacionados à qualidade em serviço que atende à saúde da mulher.

As entrevistas foram gravadas e transcritas integralmente. A coleta de dados iniciou-se em agosto e foi finalizada em setembro de 2006.

No presente trabalho, foram analisados os dados que emergiram da questão que tratava das características relacionadas à qualidade em serviços que atendem à saúde da mulher e do diálogo estabelecido entre entrevistador e sujeito. Adotou-se, no tratamento dos dados, a técnica de análise de conteúdo. Como descrito por Gaske$11^{30}$, foi elaborada uma matriz para estruturação das informações, agregando os temas das respostas em categorias definidas segundo os objetivos da pesquisa. Usaram-se, assim, as categorias de avaliação dos serviços de saúde mencionadas por Donabedian (estrutura, processos e resultados). Tal definição mostrou-se pertinente ao discurso e à prática vivenciada pelos sujeitos, os quais abordaram nas falas as características de um serviço de qualidade.

A pesquisa foi aprovada no Comitê de Ética e Pesquisa em Seres Humanos da Faculdade de Ciências da Saúde da Universidade de Brasília.

\section{Resultados}

\section{Descrição das características sociodemográficas}

Dos nove entrevistados, oito eram mulheres. Quanto à formação básica, quatro eram enfermeiros, três médicos, um farmacêutico e um odontólogo. Quanto ao tempo médio de atuação na área, os técnicos de Visa têm três anos, os coordenadores de Visa têm quatro anos e os CSM, vinte anos.

\section{Categorização das respostas sobre as características relacionadas à qualidade em serviços que atendem à saúde da mulher}

Analisando as entrevistas, identificamos que a maior parte das falas continha referências à categoria "estrutura", enquanto referências à categoria "resultados" foram menos relatadas.

Os entrevistados com a função "responsáveis pela Visa em serviços de saúde” abordaram assuntos como materiais, medicamentos e equipamentos necessários à assistência, além da multidisciplinaridade e capacitação técnica dos profissionais. Quanto aos processos, reportaram- 
se ao acolhimento e à orientação fornecida no serviço. Na categoria resultados, foi mencionada apenas a questão da resolutividade do serviço. Esse cenário pode ser mais bem compreendido por meio de trechos das verbalizações dos entrevistados, apresentados a seguir. Destaca-se que a numeração ao final da menção refere-se ao código destinado a cada entrevista.

Bom, basicamente é... material, equipamento adequado, toda essa parte de insumo, medicamento, todo o material descartável, tudo que é necessário pra ser utilizado nos exames, nas consultas (E1).

Ele tem que se adequar fisicamente, mas tem que se adequar também tecnicamente (E4).

No minimo, poder dar possibilidade de profissionais da área de ginecologia, de enfermagem, de clínica geral, com uma abordagem de psicologia, psiquiatria... Enfim, mais qualificada, com preparo, com treinamento pra isso (E7).

... depois o acolhimento, essa orientação toda pra dar embasamento ao trabalho (E1).

E até a resolutividade também. Porque muitas vezes os exames saem sem resultados, ou que se tem notícia com muito atraso e muitas vezes um diagnóstico demorado (E1).

Os entrevistados com a função de "técnicos da Visa" apresentaram a importância da privacidade e de profissionais sensibilizados para a assistência, além de se referirem às necessidades de estrutura física adequada e de materiais disponíveis. No que se refere aos processos de trabalho, destacou-se o levantamento do perfil populacional para a definição de prioridades, conforme exemplos a seguir. Não houve menção que se enquadrasse na categoria resultados.

Tem que ter uma estrutura física adequada, tem que ter uma privacidade no atendimento à mulher, tem que ter profissionais qualificados e materiais disponiveis pra fazer todo o atendimento (E2).

Profissionais sensibilizados que possam olhar a mulher como um ser integral, em todos os seus aspectos e peculiaridades (E8).

Eu acho que basicamente é conhecer as necessidades daquela comunidade e atuar em cima das prioridades pra você atender as necessidades dessas mulheres. Conhecer o perfil e necessidades daquela população e intervir de acordo com o diagnóstico (E5).

$\mathrm{Na}$ categorização das falas dos CSM, a preocupação com os processos esteve mais presente. Quanto à estrutura, além dos pontos já tratados, surgiu o tema da estruturação da rede assistencial. Em relação à categoria processos, apareceu novamente o tópico de avaliação das necessidades da população e de acolhimento. Surge aqui, pela primeira vez, a referência à importância que o diálogo com a população deveria merecer. Foram detalhados, também, aspectos mais vinculados a procedimentos, como pode ser observado nas falas. Por fim, no campo de resultados houve menção à resolutividade que o serviço proporciona.

... e ele tem que ter suporte de apoio em todos os niveis, apoio laboratorial, apoio em todos os niveis. Tem que ter um sistema de referência também bem articulado (E6).

... que se saiba encaminhar os casos alterados, para que se faça, pelo menos, uma palpação manual de mamas e que se saiba encaminhar os casos alterados (E7).

E, claro, a orientação quanto ao planejamento familiar e a possibilidade de oferecimento dos métodos (E7).

E ele tem que ser resolutivo, tem que resolver grande parte dos problemas da população. Não pode ser um serviço de triagem apenas (E6).

\section{Discussão}

O presente estudo teve como limitação a utilização de amostra de conveniência, ou seja, que não é representativa da população que se pretendeu estudar. A limitação apontada não prejudica o objetivo do trabalho, que, por se tratar de uma pesquisa exploratória, buscou explicitar e compreender as diferentes visões das características relacionadas à qualidade em serviços que atendem à saúde da mulher.

Em relação aos trabalhadores de Visa, o tempo de experiência na área foi semelhante ao perfil apresentado no Censo Nacional dos Trabalhadores da Vigilância Sanitária de 2004. Neste censo, existiam 32.037 profissionais atuando na área, sendo $60 \%$ lotados nos municípios e $60 \%$ com cinco anos ou menos de experiência na $\mathrm{Visa}^{31}$. Em contraposição, os entrevistados da área de saúde da mulher apresentaram mais experiência na sua área de atuação, pois dois sujeitos da pesquisa desenvolvem atividades relacionadas à saúde da mulher desde a residência médica.

Mesmo quando utilizados outros modelos teóricos de avaliação da qualidade, a categorização de falas de entrevistados em torno da tríade de Donabedian (estrutura-processo-resultado) tem sido importante instrumento analítico. Essa possibilidade facilitou a visualização das questões apresentadas pelos sujeitos de pesquisa, sem desconsiderar a subjetividade inerente aos dados qualitativos. 
$\mathrm{Na}$ análise dos trechos mencionados pelos participantes, é predominante o olhar da qualidade pela avaliação da estrutura dos serviços. No campo da Visa, o olhar direcionado à estrutura configura-se em marca tradicional, principalmente quando se refere às inspeções ${ }^{24}$. Dentre os aspectos de estrutura física, foram citadas questões relativas a medicamentos, produtos e recursos humanos.

Os processos de trabalho, citados principalmente pelo grupo de CSM, revelaram a preocupação com tópicos fundamentais, como conhecimento das necessidades e diálogo com a população; além de acolhimento, ponto relevante para a concretização da humanização dos cuidados em saúde.

Contudo, neste estudo, as falas dos pesquisados que pudessem ser categorizadas como resultados limitaram-se à citação sobre a resolutividade dos serviços. Não houve nas entrevistas a menção a abordagens mais ampliadas que vislumbrassem melhoria para a saúde da população, como o monitoramento por indicadores, a satisfação dos clientes ou o alívio de sofrimento.

Estudos sobre resultados focalizando a satisfação dos usuários são cada vez mais frequentes, já que estamos nos afastando do modelo médico-centrado para um paradigma em que a tomada de decisão é conjunta e informada ${ }^{32}$. Ressalta-se, também, a importância do uso de sistemas de informações gerenciais, citado por Cam$\operatorname{pos}^{25}$ como significante fonte para construção de indicadores de resultados na avaliação da qualidade da atenção primária à saúde.

No contexto da avaliação de resultados de serviços de saúde, podemos citar o Sistema de Informação da Atenção Básica (SIAB), Sistema de Informações Ambulatoriais do Sistema Único de Saúde (SIA/SUS) e o Sistema de Informações Hospitalares do SUS (SIH/SUS) como importantes subsídios à avaliação e formulação de estratégias.

A observação de que a estrutura e o processo são os pontos mais lembrados pelos agentes de Visa e CSM, na compreensão de quais seriam as características relativas à qualidade de um serviço de saúde, aponta para possível prejuízo na avaliação por resultados. Isso pode trazer restrições às análises efetuadas por esses atores, já que a totalidade da avaliação não pode ser alcançada sem uma cuidadosa abordagem dos resultados da atenção.

Mesmo com o entendimento da complexidade do tema e a subjetividade em torno do assunto qualidade, com possibilidade de uso de múltiplos caminhos metodológicos ${ }^{33}$, a crescen- te incorporação de tecnologias na saúde nos impõe a reflexão sobre as seguintes questões: a avaliação da qualidade que considera apenas estrutura e processos conseguirá identificar os reais benefícios trazidos pelo acréscimo de novas tecnologias? Será possível avaliar o risco/benefício dos serviços sem conhecer efetivamente seu impacto na saúde da população?

O documento da Política de Atenção Integral à Saúde da Mulher ${ }^{19}$ reforça que $A$ humanização e a qualidade da atenção em saúde são condições essenciais para que as ações de saúde se traduzam na resolução dos problemas identiûcados, na satisfação das usuárias, no fortalecimento da capacidade das mulheres frente à identiûcação de suas demandas, no reconhecimento e reivindicação de seus direitos e na promoção do autocuidado.

Adami e Maranhão ${ }^{34}$ ressaltam a importância de profissionais de saúde apreenderem a concepção de avaliação da qualidade, a fim de integrarem métodos para promoção e manutenção da qualidade requerida no contexto dos serviços. Para as autoras, a monitorização da qualidade, a partir da identificação e da correção precoce dos desvios encontrados, deve ser efetuada sob uma ótica pedagógica, em sobreposição à visão punitiva. Para tanto, a capacitação dos profissionais de saúde é condição essencial.

Como já identificado em outro estudo, torna-se imperativo adotar estratégias de capacitação e fortalecimento da própria Visa como área de produção do saber e de utilização de novos instrumentos de avaliação da qualidade dos serviços $^{24}$. Entre eles, podem ser mencionados o uso da epidemiologia e o da educação em saúde ${ }^{24}$. Isso não exclui a necessária formação de demais gestores da saúde, como os coordenadores de saúde da mulher, para o uso de indicadores epidemiológicos como subsídio à avaliação dos resultados dos processos assistenciais.

Ressalta-se que a incorporação da cultura do planejamento no campo da saúde requer a construção, a validação e a incorporação de indicadores capazes de mensurar o impacto das ações realizadas, o que ainda se mostra incipiente no Brasil.

É fundamental, por fim, também a reflexão sobre a importância da integração de processos avaliativos e de proposição de mudanças por parte da Visa e das áreas assistenciais, possibilitando a busca de objetivos comuns e a potencialização dos projetos desenvolvidos pelas secretarias de saúde.

Recomenda-se a ampliação de estudos nessa área, com a incorporação de dados coletados 
com prestadores do cuidado e as usuárias do serviço - na perspectiva da efetividade psicossocial, condizente com a ideia de que o processo avaliativo pressupõe a negociação entre os atores envolvidos.

\section{Colaboradores}

CS Maia trabalhou na concepção, coleta e interpretação dos dados e na redação do artigo; DRC Freitas, na interpretação dos dados e na redação; D Guilhem, na concepção e revisão crítica do artigo; AF Azevedo, na interpretação dos dados e na revisão crítica do artigo.

\section{Referências}

1. Costa AC, Rozenfeld S. Constituição da vigilância sanitária no Brasil. In: Rozenfeld S, organizador. Fundamentos da vigilância sanitária. Rio de Janeiro: Editora Fiocruz; 2000. p. 15-40.

2. Lucchese G. Globalização e regulação sanitária: os rumos da vigilância sanitária no Brasil [tese]. Rio de Janeiro: Ensp/Fiocruz; 2001.

3. Costa EA. Elementos teóricos para o estudo da vigilância sanitária. In: Costa EA, organizador. Vigilância sanitária: proteção e defesa da saúde. São Paulo: Sobravime; 2004. p. 68-98.

4. Brasil. Lei $\mathrm{n}^{\circ} 8.080$, de 19 de setembro de 1990. Dispõe sobre as condições para a promoção, proteção e recuperação da saúde, a organização e o funcionamento dos serviços correspondentes e dá outras providências. Diário Oficial da União 1990; 19 set.

5. Eduardo MBP. Vigilância sanitária. São Paulo: Faculdade de Saúde Pública da Universidade de São Paulo; 1998. (Série Saúde \& Cidadania).

6. Brasil. Ministério da Saúde. Direito sanitário e saúde pública. Brasília: Ministério da Saúde; 2003.

7. Brasil. Agência Nacional de Vigilância Sanitária (Anvisa). Pediatria: prevenção e controle de infeção hospitalar. Brasília: Anvisa; 2005.

8. Brasil. Agência Nacional de Vigilância Sanitária (Anvisa). PNASS - Programa Nacional de Avaliação de Serviços de Saúde: resultado do processo avaliativo 2004-2006. Brasília: Anvisa; 2007.

9. Henriques CMP. A vigilância e a reforma sanitária: quatro passos. Gestão médica 2006; 32-34.

10. Uchimura KY, Bosi MLM. Qualidade e subjetividade na avaliação de programas e serviços em saúde. Cad Saude Publica 2002; 18(6):1561-1569.

11. Gastal FL, Roessler IF. Talsa multiplicadores: avaliação e qualidade (módulo 2). [documento na Internet]. 2006[acessado 2006 jun 4]. Disponível em: http://www.onaeducare.org.br

12. Azevedo CA. Avaliação de desempenho de serviços de saúde. Rev Saude Publica 1991; 25:64-71.

13. Donabedian A. The seven pillars of quality. Arch Pathol Lab Med 1990; 114:1115-1119.

14. Aguilar MJ, Ander-Egg E. Avaliação de serviços e programas sociais. Petrópolis: Vozes; 1994.

15. Donabedian A. Quality assessment an assurance: unity of purpose, diversity of means. Inquiry 1988; 25:173-192. 
16. Novaes HMD. Avaliação de programas, serviços e tecnologias em saúde. Rev Saude Publica 2000; 34:547-549.

17. World Health Organization Europe. What are the advantages and limitations of different quality and safety tools for health care? Copenhagen: WHO Regional Office for Europe; 2005.

18. Costa AM, Guilhem D, Walter MIMT. Atendimento a gestantes no Sistema Único de Saúde. Rev Saude Publica 2005; 39(5):768-774.

19. Brasil. Ministério da Saúde. Política Nacional da Atenção Integral à Saúde da Mulher: princípios e diretrizes. Brasília: Ministério da Saúde; 2004.

20. Brasil. Ministério da Saúde. Área técnica de saúde da mulher. Brasília: Ministério da Saúde; 2003.

21. Oba MDV, Tavares MSG. Aspectos positivos e negativos da assistência pré-natal no município de Ribeirão Preto. SP. Rev Latino-Am Enfermagem 2000; 8(2):11-17.

22. Santos ALD, Rosenburg CP, Buralli KO. Histórias de perdas fetais contadas por mulheres: estudo de análise qualitativa. Rev Saude Publica 2004; 38(2):268276.

23. Melleiro MM, Gualda DMR. Experiências e expressões de gestantes na interação com o sistema de saúde: um enfoque fotoetnográfico. Rev LatinoAm Enfermagem 2004; 12(3):503-510.

24. Maia CS. Vigilância sanitária e saúde da mulher: um estudo sobre a integralidade no SUS [dissertação]. Brasília: Faculdade de Ciências da Saúde, Universidade de Brasília; 2007.

25. Campos CEA. Estratégias de avaliação e melhoria contínua da qualidade no contexto da atenção primária à saúde. Rev Bras Saúde Matern Infant 2005; 5(Supl.1):563-569.

26. Portela MC. Avaliação da qualidade em saúde. In: Rozenfeld S, organizador. Fundamentos da vigilância sanitária. Rio de Janeiro: Editora Fiocruz; 2000. p. 259-269.
27. Minayo MCS. Introdução. In: Minayo MCS, organizadora. Avaliação por triangulação de métodos: abordagem de programas sociais. Rio de Janeiro: Editora Fiocruz; 2005. p. 53-70.

28. Minayo MCS. O desafio do conhecimento: pesquisa qualitativa em saúde. $8^{\text {a }}$ ed. São Paulo: Hucitec; Rio de Janeiro: Abrasco, 2004.

29. Franco MLPB. Análise de conteúdo. 2a ed. Brasília: Líber Livro; 2005.

30. Gaskell G. Entrevistas individuais e grupais. In: Bauer MW, Gaskell G, organizadores. Pesquisa qualitativa com texto, imagem e som. Petrópolis: Vozes; 2003. p. 64-89.

31. Brasil. Agência Nacional de Vigilância Sanitária (Anvisa). Censo nacional dos trabalhadores da vigilância sanitária. Brasília: Anvisa; 2004.

32. Driel MLV, Sutter AID, Christiaens TCM, Maeseneer JMD. Quality of care: the need for medical, contextual and policy evidence in primary care. $J$ Eval in Clinical Practice 2005; 11(5):417-429.

33. Caldeira AP, Oliveira RM, Rodrigues AO. Qualidade da assistência materno-infantil em diferentes modelos de atenção primária. Cien Saude Colet 15(Supl.2):3139-3147.

34. Adami NP, Maranhão AMSA. Qualidade dos serviços de saúde: conceitos e métodos avaliativos. Acta Paul Enf 1995; 8(4):47-55.

Artigo apresentado em 21/08/2008

Aprovado em 12/11/2008

Versão final apresentada em 15/10/2008 